\title{
KID Syndrome and Hidradenitis Suppurativa: A Rare Association Responding to Surgical Treatment
}

\author{
Vincenzo Bettolia Riccardo Forconi ${ }^{a} \quad$ Ilaria Pezzini $^{b}$ Ruby Martinelloc \\ Valeria Scuderi ${ }^{a} \quad$ Piera Zedde $^{a}$ Natale Schettini ${ }^{a} \quad$ Lucrezia Pacetti $^{a}$ \\ Monica Corazza a
}

aSection of Dermatology, Department of Medical Sciences, University of Ferrara, Ferrara, Italy; bPlastic and Reconstructive Surgery, Azienda Ospedaliero-Universitaria di Ferrara, Arcispedale Sant'Anna, Ferrara, Italy; 'Department of Morphology, Surgery and Experimental Medicine, Institute of Obstetrics and Gynecology, University of Ferrara, Ferrara, Italy

\section{Established Facts}

- Keratitis-ichthyosis-deafness syndrome + hidradenitis suppurativa is a rare association and needs a complex therapeutic management.

- There is lack of information regarding the treatment in the literature due to the rarity of the association.

\section{Novel Insights}

- Surgical therapy for hidradenitis suppurativa lesions has achieved excellent results in a patient affected by this rare association.

\section{Keywords}

Keratitis-ichthyosis-deafness syndrome $\cdot$ Hidradenitis suppurativa $\cdot$ Rare · Genodermatosis · Surgical treatment

\section{Abstract \\ Background: Keratitis-ichthyosis-deafness (KID) syndrome is a rare genodermatosis characterized by keratitis, neuro- sensorial auditory impairment and ichthyosiform skin in- volvement. Frequent complications of the syndrome are chronic, opportunistic cutaneous infections, and the devel-}

opment of skin cancers. Several cases of association between KID syndrome and other conditions, including hidradenitis suppurativa (HS), are described in the literature. This correlation could be explained by the hyperproliferative state of the epidermis, which occurs in KID syndrome and may favor follicular plugging. Objectives: The aim of this study was to describe a very rare case of association between KID syndrome and HS and its complex therapeutic management. Results: The failure of the drugs commonly used in HS and the excellent results of surgery, although difficult to achieve, were experienced. Conclusion: Despite the techni- karger@karger.com www.karger.com/sad

(C) 2020 S. Karger AG, Basel

Karger ${ }^{\prime}=$
Vincenzo Bettoli

Section of Dermatology, Department of Medical Sciences Azienda Ospedaliero, Universitaria di Ferrara, Arcispedale Sant'Anna

Via Aldo Moro 8, IT-44124 Ferrara (Italy)

vincenzo.bettoli@gmail.com 
cal difficulties related to surgery, namely, cutaneous superinfections, frequent dehisce of the suture, and closure by secondary intention, the authors strongly recommend the surgical approach in these patients. $\odot 2020$ S. Karger AG, Basel

\section{Introduction}

Keratitis-ichthyosis-deafness (KID) syndrome is a rare genodermatosis that typically results from missense mutations in the GJB2 gene. This gene encodes a transmembrane, gap junction channel-forming protein named connexin 26, whose aberrant behavior causes cellular malfunction [1]. The commonest mutation discovered as a cause of KID syndrome is D50N (p.Asp50Asn). Less common mutations are G12R (p.Gly12Arg), S17F (p.Ser17Phe), D50Y (p.Asp50Tyr), I30N (p.Ile30Asn), and G11E (p.Gly11Glu) [2]. Furthermore, early lethality is associated with G45E (p.Gly45Glu) and A88V (p.Ala$88 \mathrm{Val}$ ) mutations [3]. The resulting syndrome is characterized by photophobia and ocular neovascularization (keratitis), progressively reducing visual acuity, neurosensorial auditory impairment, and skin involvement and by hyperkeratotic plaques and palmoplantar keratoderma with alopecia and/or onychodystrophy. Frequent complications of the syndrome are chronic, opportunistic cutaneous infection, and the development of skin malignancies, mostly squamous cell carcinoma [4]. KID syndrome may be associated with other conditions as described in the literature [5-7]; we present a case of association between KID syndrome and hidradenitis suppurativa (HS).

\section{Case Report}

A woman, born of non-consanguineous healthy parents, presented with congenital neurosensorial bilateral hearing loss and ichthyosiform dermopathy characterized by palmoplantar keratoderma, widespread scales, fingernail and toenail dystrophy, and a hyperkeratotic plaque of the scalp. At the age of 38 years, after genetic counseling and analysis, a KID syndrome related to the D50N mutation was diagnosed.

The patient also reported recurrent inguinal abscesses since adolescence, treated with surgical drainages. At the age of 39 years, she developed a complex inflammatory skin condition characterized by nodules, abscesses, and fistulas in the genital and groin area, diagnosed as HS. No signs of acne vulgaris or dissecting cellulitis (DC) of the scalp were found during clinical examination and no history of these conditions was reported by the patient. The physical examination showed the involvement of the right inguinal and crural region, right hemivulva, and perianal region (Hurley

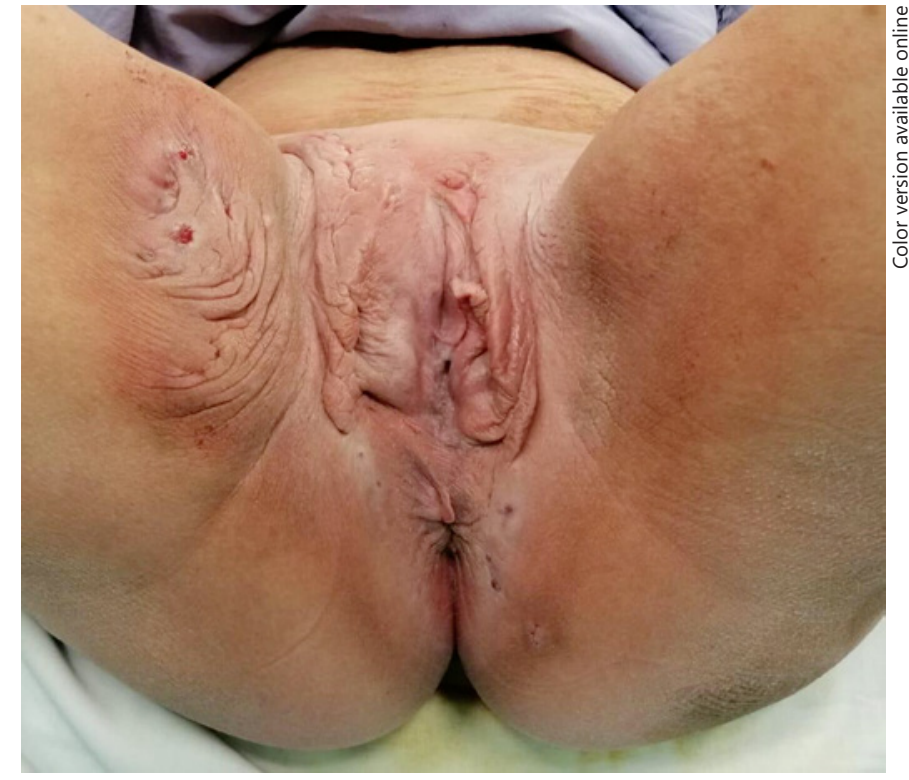

Fig. 1. Presurgical HS localizations: right inguinal and crural region, right thigh root, right hemivulva, and perianal region. HS, hidradenitis suppurativa.

III, Sartorius 76) (Fig. 1). MRI reported skin and subcutaneous edema extending from the medial region of the right thigh root to the ipsilateral vulvar region. In this context, there was evidence of a vulvar fistula that branched out into at least 3 fistulas that developed up to the ipsilateral groin region. Another tunnel from the anal canal to the skin plane was noticed. Gynecologic evaluation confirmed the absence of fistulas involving the vaginal walls. The patient was initially treated with an antibiotic therapy (combination of clindamycin and rifampicin) with limited and temporary benefit on HS. Subsequently, the patient was treated with adalimumab, which led to a mild clinical improvement but not to any benefit on the quality of life. After gynecologic and plastic surgery consultation the patient was subjected to excision of the areas affected by HS. A large soft tissue resection, including the anterior half of the large right lip and the medial part of the root of the ipsilateral thigh, was performed. The surgical procedure ended with plastic reconstruction by detaching and sliding skin flaps (Fig. 2). During the postoperative course, the patient presented with fever and slight foul-smelling suppuration on the surgical wound. Skin swabs demonstrated a pseudomonas aeruginosa infection. Targeted antibiotic therapy was effective, and the surgical wound repaired by secondary intention, with optimal surgical outcome (Fig. 3).

\section{Discussion}

HS is usually a sporadic disease but sometimes appears in the context of rare syndromes; in addition to the case we are presenting, an association with KID syndrome is 


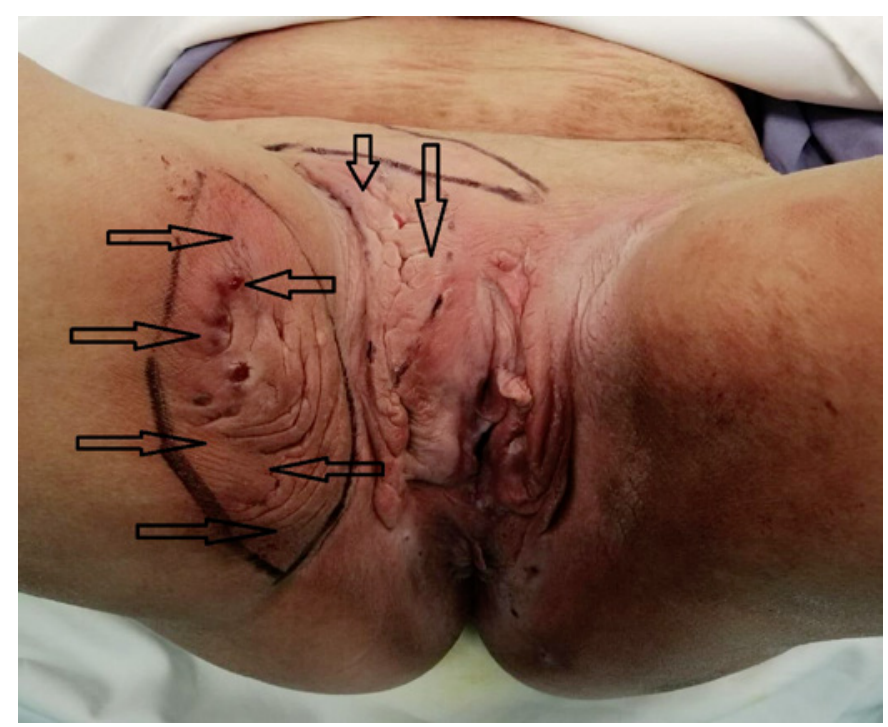

Fig. 2. Surgical strategy: the arrows start from the skin areas used for sliding the flaps and end in the portions to be repaired after excision.

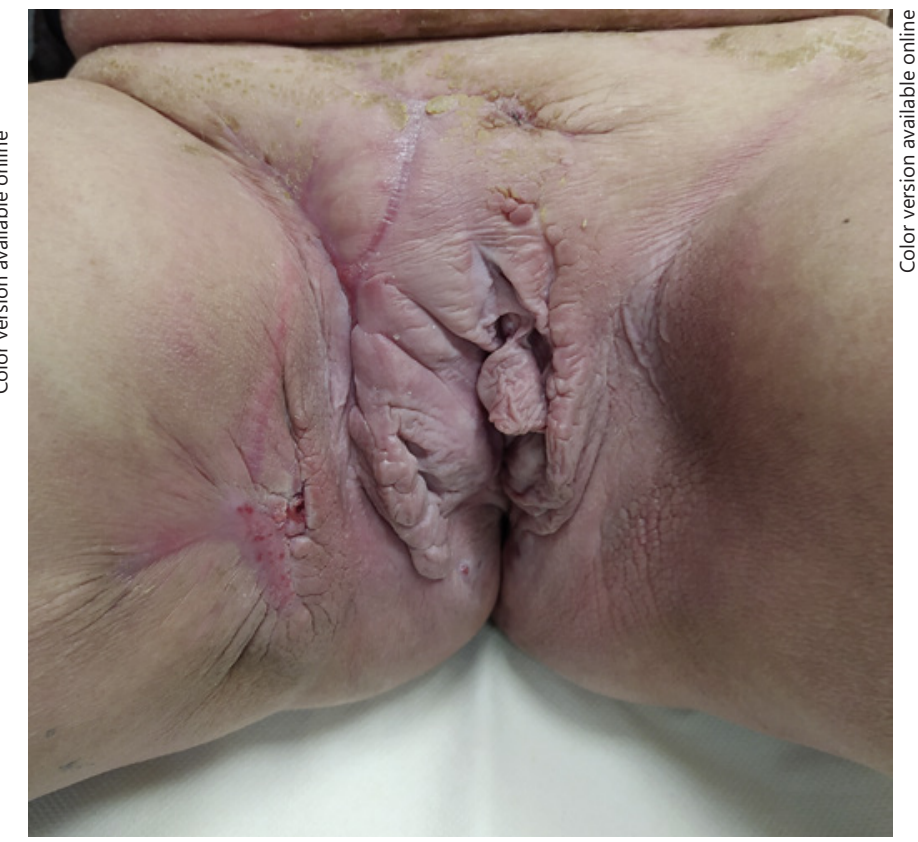

Fig. 3. Postsurgical outcome.

Table 1. KID and HS association features in the literature

\begin{tabular}{lll}
\hline Reference & Cutaneous features of KID & HS localizations \\
\hline Present case & $\begin{array}{l}\text { Widespread scales, palmoplantar keratoderma, fingernail and toenail } \\
\text { dystrophy, and a hyperkeratotic plaque of the scalp }\end{array}$ & Genitals and groins \\
\hline Maintz et al. [5] & Ichthyosis cutis, palmoplantar keratoderma toenail dystrophy & Axillae and groins, associated with AV and DC D50N \\
\hline Nyquist et al. [9] & Dry skin, palmoplantar keratoderma & Groins, associated with AV and DC \\
\hline Prasad and Bygum [10] & $\begin{array}{l}\text { Dry skin, widespread hyperkeratotic plaques, sparse hair, fingernail, } \\
\text { and toenail dystrophy }\end{array}$ & Axillae and groins, associated with AV and DC D50N \\
\hline Montgomery et al. [11] & Leukonychia, mild palmoplantar keratoderma & Axillae and groins, associated with AV and DC A40V \\
\hline Lazic et al. [2] & Unknown & Unknown, associated with AV and DC \\
\hline \multicolumn{1}{c}{ AV, acne vulgaris; DC, dissecting cellulitis; KID, keratitis-ichthyosis-deafness; HS, hidradenitis suppurativa. }
\end{tabular}

reported only in 5 cases, as part of the follicular occlusion triad (HS, acne vulgaris, and dissecting cellulitis of the scalp) [8]. In 3 of these patients D50N mutation of GJB2 was identified $[5,9,10], 1$ patient presented with A40V mutation [11], and another 1 with G12R mutation [2] (Table 1). This correlation could be explained by the hyperproliferative state of the epidermis which occurs in KID syndrome; this condition may lead to follicular plug- ging, which is widely considered a relevant factor in the pathogenesis of HS.

Surgical therapy is becoming increasingly common in the treatment of HS and the authors experienced the excellent results obtained in this case of association with KID syndrome. Nevertheless, 2 critical issues are related to the surgical procedure. The first is represented by the high risk of opportunistic cutaneous infection of the sur- 
gical site because the affected skin of the big folds in KID patients is likely to be more humid than usual and prone to overinfection. The second, related to the first one, is represented by a high risk of closure by secondary intention. To reduce the risk of surgery-related complications, it is recommended to use antiseptic dressings aimed at decreasing the surface microbial load and specific antibiotic treatment if microbial superinfection is suspected.

\section{Conclusions}

Despite the critical point previously raised, the authors strongly recommend the surgical approach in patients with this rare association. The description of this case is significant for 2 reasons. (1) The rarity of the association between KID and HS. This is the sixth case described in the literature. (2) Support of the surgical approach, despite the critical points raised.

\section{Statement of Ethics}

The patient has given her written informed consent to publish photos and details of the case.

\section{Conflict of Interest Statement}

None of the authors have any declaration of any conflict of interest to report.

\section{Funding Sources}

The authors did not receive any funding.

\section{Author Contributions}

Vincenzo Bettoli: study conception and design, general supervision of the project, revising the article, and approving the final version of submission; Riccardo Forconi: study conception and design, drafting the article, and approving the final version of submission; Ilaria Pezzini: substantial acquisition of data, revising the article, and approving the final version of submission; Ruby Martinello: substantial acquisition of data, revising the article, and approving the final version of submission; Valeria Scuderi: substantial acquisition of data, drafting the article, providing writing assistance, and approving the final version of submission; Piera Zedde: collection of data, analysis and interpretation of data, providing writing assistance, and approving the final version of submission; Natale Schettini: collection of data, providing care for the study patient, revising the paper, and approving the final version of submission; Lucrezia Pacetti: providing care for the study patient, collection of data, providing writing assistance, and approving the final version of submission; Monica Corazza: study conception and design, general supervision of the research group, revising the article, and approving the final version of submission.

\section{References}

1 Lee MY, Wang HZ, White TW, Brooks T, Pittman A, Halai $\mathrm{H}$, et al. Allele-specific small interfering RNA corrects aberrant cellular phenotype in keratitis-ichthyosis-deafness syndrome keratinocytes. J Invest Dermatol. 2020 May;140(5):1035-44.e7.

2 Lazic T, Li Q, Frank M, Uitto J, Zhou LH. Extending the phenotypic spectrum of keratitisichthyosis-deafness syndrome: report of a patient with GJB2 (G12R) connexin 26 mutation and unusual clinical findings. Pediatr Dermatol. 2012;29(3):349-57.

3 Lilly E, Bunick CG, Maley AM, Zhang S, Spraker MK, Theos AJ, et al. More than keratitis, ichthyosis, and deafness: multisystem effects of lethal GJB2 mutations. J Am Acad Dermatol. 2019;80(3):617-25.

4 Mazereeuw-Hautier J, Bitoun E, ChevrantBreton J, Man SY, Bodemer C, Prins C, et al. Keratitis-ichthyosis-deafness syndrome: disease expression and spectrum of connexin 26 (GJB2) mutations in 14 patients. Br J Dermatol. 2007;156(5):1015-9.
5 Maintz L, Betz RC, Allam JP, Wenzel J, Jaksche A, Friedrichs N, et al. Keratitis-ichthyosis-deafness syndrome in association with follicular occlusion triad. Eur J Dermatol. 2005; 15(5):347-52.

6 Fozza C, Poddie F, Contini S, Galleu A, Cottoni F, Longinotti M, et al. Keratitis-ichthyosis-deafness syndrome, atypical connexin GJB2 gene mutation, and peripheral T-cell lymphoma: more than a random association? Case Rep Hematol. 2011;2011:848461.

7 Salman A, Gencosmanoglu DS, Yucelten AD, Elcioglu N, Richard G, Demirkesen C. Successful treatment of pityriasis lichenoides chronica with narrow-band ultraviolet $B$ therapy in a patient with keratitis-ichthyosisdeafness syndrome: a case report. Dermatol Online J. 2016;22(5):13030/qt3sd5h1n2.
8 Gasparic J, Theut Riis P, Jemec GB. Recognizing syndromic hidradenitis suppurativa: a review of the literature. J Eur Acad Dermatol Venereol. 2017;31(11):1809-16.

9 Nyquist GG, Mumm C, Grau R, Crowson AN, Shurman DL, Benedetto P, et al. Malignant proliferating pilar tumors arising in KID syndrome: a report of two patients. Am J Med Genet A. 2007;143A(7):734-41.

10 Prasad SC, Bygum A. Successful treatment with alitretinoin of dissecting cellulitis of the scalp in keratitis-ichthyosis-deafness syndrome. Acta Derm Venereol. 2013;93(4): 473-4.

11 Montgomery JR, White TW, Martin BL, Turner ML, Holland SM. A novel connexin 26 gene mutation associated with features of the keratitis-ichthyosis-deafness syndrome and the follicular occlusion triad. J Am Acad Dermatol. 2004;51(3):377-82. 